\title{
Radioembolization versus chemoembolization for unresectable hepatocellular carcinoma: a meta-analysis of randomized trials
}

This article was published in the following Dove Press journal: OncoTargets and Therapy

\author{
Andrea Casadei Gardini',* \\ Emiliano Tamburini2,* \\ Mercedes Iñarrairaegui ${ }^{3}$ \\ Giovanni Luca Frassineti ${ }^{1}$ \\ Bruno Sangro 3 \\ 'Department of Medical Oncology, \\ Istituto Scientifico Romagnolo per \\ lo Studio e Cura dei Tumori (IRST) \\ IRCCS, Meldola, Italy; ${ }^{2}$ Department of \\ Medical Oncology, Ospedale Infermi, \\ Rimini, Italy; ${ }^{3}$ Liver Unit, Clínica \\ Universidad de Navarra-IDISNA and \\ CIBEREHD, Pamplona, Spain \\ *These authors contributed equally \\ to this work
}

Purpose: This study aimed to compare clinically relevant outcomes following transarterial chemoembolization (TACE) and transarterial radioembolization (TARE) in patients with unresectable hepatocellular carcinoma (HCC) using only prospective randomized clinical trials as a source of information.

Materials and methods: A meta-analysis was performed to compare the efficacy of TARE and TACE in treating patients with unresectable HCC. Only prospective randomized trials were included in the quantitative analysis. Overall and progression-free survival, disease control rate, and transplantation rate were the variables under analysis.

Results: Overall survival at 1 year was similar between the two treatment groups $(\mathrm{OR}=1.31$, 95\% CI: $0.56-3.04, P=0.53)$. Progression-free survival at 1 year was also not statistically different between the two treatments $(\mathrm{OR}=0.23,95 \% \mathrm{CI}$ : $0.02-2.45, P=0.22)$. Although a higher proportion of patients underwent transplantation in the TARE group (30\% vs $20.8 \%$ ), this difference was not statistically significant ( $\mathrm{OR}=0.68,95 \% \mathrm{CI}: 0.23-2.01 ; P=0.49)$.

Conclusion: TARE and TACE provide similar outcomes in unresectable HCC. The role of TARE should be explored in selected patient subpopulations in future clinical trials.

Keywords: selective internal radiation, SIRT, TARE, TACE, outcome, transplantation rates

\section{Introduction}

Transarterial chemoembolization (TACE) is the most widely used primary treatment for hepatocellular carcinoma (HCC). ${ }^{1}$ According to Western guidelines and clinical practice, ${ }^{2,3}$ as well as based on positive results from two randomized trials and three subsequent meta-analyses, it is the recommended first-line therapy for those patients who are in the intermediate stage and for those who are in the early stage but cannot be treated by resection, transplantation, or percutaneous ablation. ${ }^{4}$ Conventional TACE consists of the intra-arterial infusion of a cytotoxic agent usually mixed with the oily contrast medium Lipiodol, followed by embolization of the tumor blood vessels with gelfoam or calibrated particles, while in TACE based on drug-eluting beads (DEBs), ie, DEB-TACE, DEBs loaded with doxorubicin are used. ${ }^{5}$ Both procedures rely on the combined cytotoxic and ischemic effect to induce tumor cell killing.

In contrast, transarterial radioembolization (TARE, also called selective internal radiation therapy or SIRT) consists of the intra-arterial infusion of much smaller beads that are loaded with a radioactive isotope (yttrium-90), and it relies on the beta radiation emitted by the isotope to induce tumor necrosis..$^{5}$ In noncontrolled prospective and retrospective series, TARE resulted in high rates of objective tumor responses,
Correspondence: Bruno Sangro Liver Unit, Clínica Universidad de Navarra, Pio XII 36, 31008 Pamplona, Spain

Tel +3494825 5400

Fax +34948296500

Email bsangro@unav.es 
prolonged time to progression, and overall survival similar to those with TACE. ${ }^{5}$ Over the past few years, three prospective randomized clinical trials have compared both intra-arterial procedures with main end points other than overall survival. ${ }^{6,7}$ However, survival outcomes have also been reported in these three trials. We have performed a meta-analysis to compare the efficacy of SIRT and TACE in treating patients with unresectable HCC considering only randomized studies that may provide the highest possible level of evidence.

\section{Materials and methods}

\section{Study design and inclusion criteria}

Clinical trials comparing TACE and SIRT were searched in PubMed. Only randomized controlled trials that included patients with $\mathrm{HCC}$ were considered eligible and included in the quantitative analysis. A primary analysis was planned to compare overall survival between TACE and SIRT, while progression-free survival, disease control rate, progression rate, and rate of liver transplantation were secondary aims.

\section{Search strategy}

Figure 1 reports the search strategy followed in this meta-analysis. A bibliographic research was conducted of the PubMed, Cochrane Library, and Embase databases. Keywords used included chemoembolization/TACE AND radioembolization/ TARE/SIRT/yttrium-90 AND hepatocellular carcinoma. Articles published in English until April 2017 were retrieved. Relevant reviews and meta-analyses of locoregional treatments in unresectable HCC were also examined for potential suitable studies and data. The proceedings of the annual meetings of the American Society of Clinical Oncology (ASCO

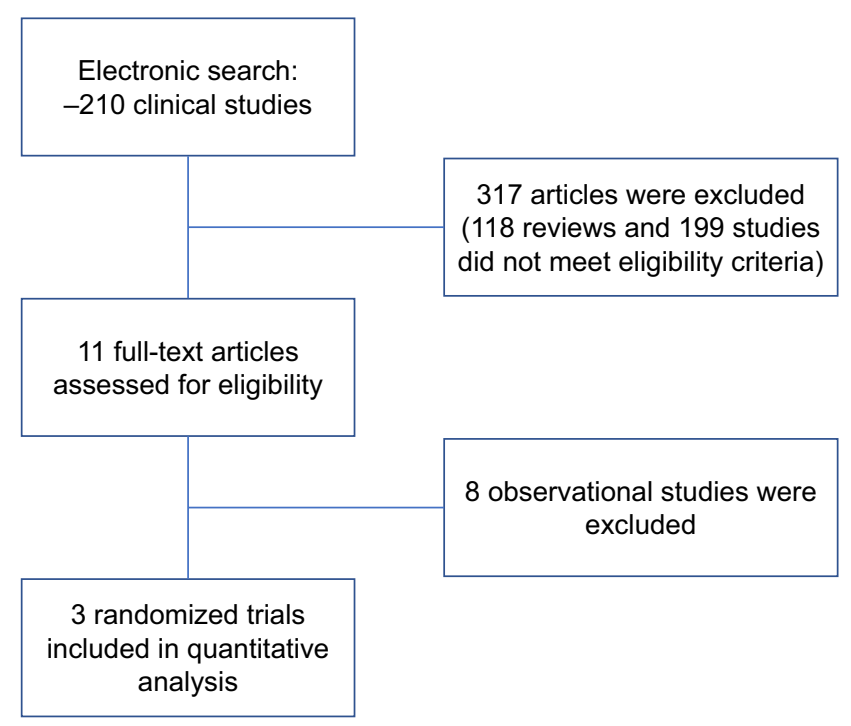

Figure I Process of selection of studies for the meta-analysis. and the ASCO gastrointestinal [ASCO GI] Cancers Symposium), European Society of Clinical Oncology (ESMO and ESMO GI), European Association for the Study of the Liver (EASL), American Association for the Study of Liver Diseases (AASLD), and International Liver Cancer Association (ILCA) in the years 2010 through 2017 were systematically reviewed to detected unpublished data if pertinent.

\section{Data extraction and management}

Two authors (ACG and GLF) independently screened the titles and abstracts of all the selected studies. All the abstracts of potentially eligible trials were independently read by the same authors who decided whether the study was selected. The full text of all selected papers was then analyzed by the same authors to select all the trials finally included in the pooled analysis. When there are discrepancies in trial search or selection, they discussed with a third researcher (BS) to reach a final consensus. The internal validity of the trial was assessed by evaluating the method used for randomization, blindness, report of missing data, allocation sequence, and allocation concealment. All selected trials published as fulltext articles in a peer-reviewed journal were analyzed and classified using the Jadad score when possible. Qualitative and quantitative analyses of the selected articles were independently performed by the same two authors (ACG and GLF); when there are discrepancies, they discussed with a third researcher (BS) to reach a final consensus. Overall and progression-free survival, disease control rate, and transplantation rate were the variables under analysis.

\section{Statistical analysis}

Meta-analysis was performed in accordance with the PRISMA statement recommendations. ${ }^{9}$ Data were entered in a computer database for transfer and statistical analysis in Review Manager 5.2. Heterogeneity among the trials was assessed with descriptive aim using the $I^{2}$ test. $I^{2}$ values above $50 \%$ were deemed to suggest large among-trial heterogeneity, values of $25 \%-50 \%$ were deemed to show modest heterogeneity, and values below $25 \%$ were deemed to represent low heterogeneity. A level $<5 \%$ was assumed to be statistically significant. Differences between categorical outcome parameters were quantified using the OR and corresponding 95\% 95\% CI. Summary statistics for dichotomous outcome data were assessed using the Mantel-Haenszel method. Summary statistics for generic inverse variance data were calculated using the inverse variance method. Pooled analysis of the OR was performed using a random-effect model, assuming an error of $5 \%$ as an index of statistical significance. 


\section{Results}

\section{Study selection and characteristics}

The combined search yielded 67 potentially relevant articles, 64 of which were excluded because they were not randomized controlled trials. Three studies published from 2014 to 2016 were analyzed. They included 49 HCC patients treated with TARE and 48 who underwent TACE. ${ }^{6-8}$ One trial had time to progression as the primary end point and the preplanned sample size of 45 patients was not met due to slow recruitment. ${ }^{6}$ A second trial had health-related quality of life (HRQoL) as the primary end point and recruited the planned sample of 25 patients. ${ }^{7}$ The third trial was considered a pilot trial with no specific end point or sample size calculation and recruited 28 patients. ${ }^{8}$ The first two studies were judged to be of high quality and the last one was considered of moderate quality. Table 1 presents the characteristics of the patients in the three randomized clinical trials. Figure S1 shows a funnel plot.

\section{Outcomes}

A significant heterogeneity between the trials was detected for progression-free survival ( $I^{2}$ test: $76 \%$ ), while no heterogeneity was detected for overall survival, disease control rate, or transplantation rate. As illustrated in Figure 2A, there were no differences in overall survival at 1 year between the two treatment groups $(\mathrm{OR}=1.31,95 \%$
CI: $0.56-3.04, P=0.53)$. Progression-free survival at 1 year was also not statistically different between the two treatments (OR $=0.23,95 \%$ CI: $0.02-2.45, P=0.22)$, as shown in Figure 2B. Figure 3 shows how progression rates and disease control rates were also not significantly different between groups, with OR values of 0.61 (95\% CI: 0.14-2.70, $P=0.51$ ) for progression rates and 1.80 (95\% CI: 0.51-6.30, $P=0.36$ ) for disease control rates. Finally, although a higher proportion of patients underwent transplantation in the TARE group (30\% vs $20.8 \%$ ), such difference was not statistically significant ( $\mathrm{OR}=0.6895 \% \mathrm{CI}$ : $0.23-2.01, P=0.49$ ), as shown in Figure 4.

\section{Discussion}

TACE is recommended for patients with intermediate-stage HCC according to the Barcelona Clinic Liver Cancer (BCLC) classification, ${ }^{10}$ as well as for those with early-stage tumors that cannot be treated with percutaneous ablation, resection, or transplantation. ${ }^{1,2}$ This recommendation is based on strong scientific evidence coming from two randomized clinical trials in which carefully selected patients with low tumor burden were recruited. ${ }^{11,12}$ Without further scientific support, its use has nevertheless been expanded to treat almost any patient with liver-only or liver-predominant disease even in the presence of vascular invasion. ${ }^{13,14}$ In consequence, TACE is the most frequently used primary therapy for HCC worldwide. ${ }^{15}$

Table I Patient characteristics in the three randomized clinical trials

\begin{tabular}{|c|c|c|c|c|c|c|c|}
\hline \multirow{2}{*}{$\begin{array}{l}\text { Trial } \\
\text { Group }\end{array}$} & & \multicolumn{2}{|l|}{ SIRTACE ${ }^{6}$} & \multicolumn{2}{|l|}{ Mainz ${ }^{7}$} & \multicolumn{2}{|l|}{ PREMIERE $^{8}$} \\
\hline & & $\begin{array}{l}\text { TARE } \\
\mathrm{n}=13\end{array}$ & $\begin{array}{l}\text { TACE } \\
\mathrm{n}=15\end{array}$ & $\begin{array}{l}\text { TARE } \\
n=12\end{array}$ & $\begin{array}{l}\text { TACE } \\
n=12\end{array}$ & $\begin{array}{l}\text { TARE } \\
n=24\end{array}$ & $\begin{array}{l}\text { TACE } \\
n=2 \text { I }\end{array}$ \\
\hline Age, years & & 65.8 & 66.7 & 71.8 & 70.5 & 62 & 64 \\
\hline Males, \% & & 84.6 & 86.7 & 83.4 & 75 & 71 & 76 \\
\hline ECOG, n (\%) & $\begin{array}{l}0 \\
1\end{array}$ & $\begin{array}{l}10(76.9) \\
3(23.1)\end{array}$ & $\begin{array}{l}12(80.0) \\
3(20.0)\end{array}$ & $\begin{array}{l}12(100) \\
0(0)\end{array}$ & $\begin{array}{l}12(100) \\
0(0)\end{array}$ & $\begin{array}{l}24(100) \\
0(0)\end{array}$ & $\begin{array}{l}21(100) \\
0(0)\end{array}$ \\
\hline Child-Pugh class, n (\%) & $\begin{array}{l}\text { A } \\
\text { B }\end{array}$ & $\begin{array}{l}\text { I2 (92.3) } \\
\text { I (7.7) }\end{array}$ & $\begin{array}{l}13(86.6) \\
2(13.4)\end{array}$ & $\begin{array}{l}10(83.3) \\
2(16.7)\end{array}$ & $\begin{array}{l}9(75) \\
3(25)\end{array}$ & $\begin{array}{l}18(75) \\
6(25)\end{array}$ & $\begin{array}{l}17(81) \\
4(19)\end{array}$ \\
\hline BCLC stage, n (\%) & $\begin{array}{l}A \\
B \\
C\end{array}$ & $\begin{array}{l}5(38.4) \\
5(38.4) \\
3(23.0)\end{array}$ & $\begin{array}{l}4(26.6) \\
8(53.3) \\
3(20.0)\end{array}$ & $\begin{array}{l}13(86.6) \\
2(13.4) \\
0(0)\end{array}$ & $\begin{array}{l}10(83.3) \\
2(16.7) \\
0(0)\end{array}$ & $\begin{array}{l}18(75) \\
6(25) \\
0(0)\end{array}$ & $\begin{array}{l}17(8 \mathrm{I}) \\
4(19) \\
0(0)\end{array}$ \\
\hline Bilobar disease & & ND & ND & $8(67)$ & $7(58)$ & $7(29)$ & $7(33)$ \\
\hline Tumor size, mm & & ND & ND & $61.3(36.4)^{\mathrm{a}}$ & $60.8(37.6)^{\mathrm{a}}$ & $32(27-37)$ & $30(23-36)$ \\
\hline Tumor volume, $\mathrm{mL}$ & & I $37.7(237.6)^{\mathrm{a}}$ & $235.6(349.4)^{a}$ & ND & ND & ND & ND \\
\hline Total bilirubin (mg/dL) & & $1.00(0.60)^{\mathrm{a}}$ & I.08 $(0.45)^{\mathrm{a}}$ & $1.17(0.38-2.10)$ & $1.26(0.59-2.04)$ & $1.3(1.2-1.7)$ & $0.9(0.8-1.5)$ \\
\hline Albumin (g/L) & & $36.3(3.9)^{\mathrm{a}}$ & $42.0(8.0)^{\mathrm{a}}$ & $34.1(28-43)$ & $31.9(24-39)$ & $31(27-33)$ & $32(29-34)$ \\
\hline $\operatorname{AFP}(\mathrm{ng} / \mathrm{mL})$ & & $636.0(2,171.8)^{a}$ & $2,624.7(9,525.3)^{\mathrm{a}}$ & I4.0 (6.2-32,346) & $7.8(2.7-1,847)$ & $\begin{array}{l}<200: 88 \% \\
>200: 10 \%\end{array}$ & $\begin{array}{l}<200: 90 \% \\
>200: 12 \%\end{array}$ \\
\hline
\end{tabular}

Notes: aMean (SD). Values are expressed as median (IQR) unless otherwise indicated. Data from Salem R et al; Pitton MB; Kolligs FT.6-8

Abbreviations: AFP, alpha-fetoprotein; BCLC, Barcelona Clinic Liver Cancer; ECOG, Eastern Cooperative Oncology Group; ND, not determined; TACE, transarterial chemoembolization; TARE, transarterial radioembolization. 


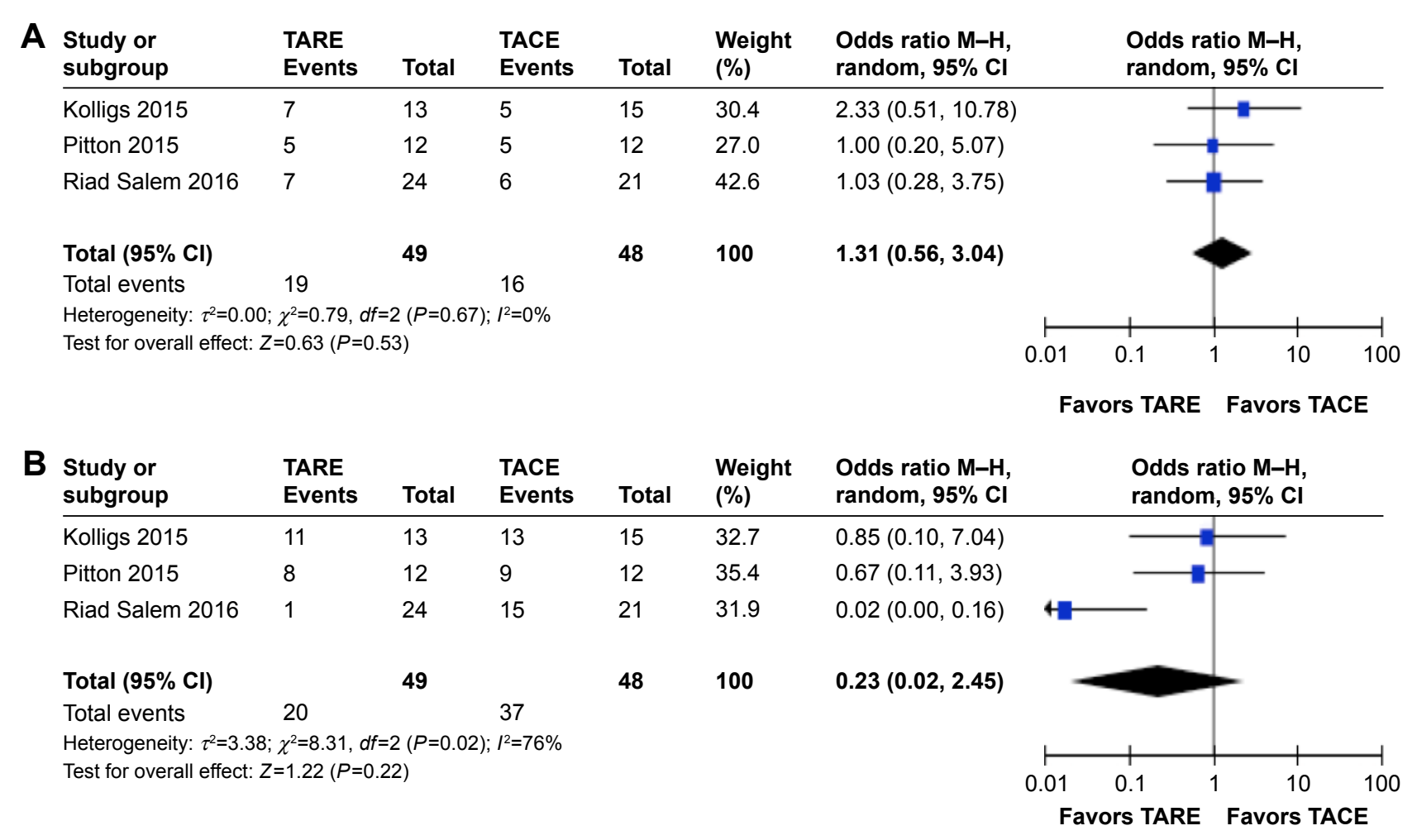

Figure 2 Forest plots of I-year survival rate (A) and I-year progression-free survival (B).

Abbreviations: TACE, transarterial chemoembolization; TARE, transarterial radioembolization.

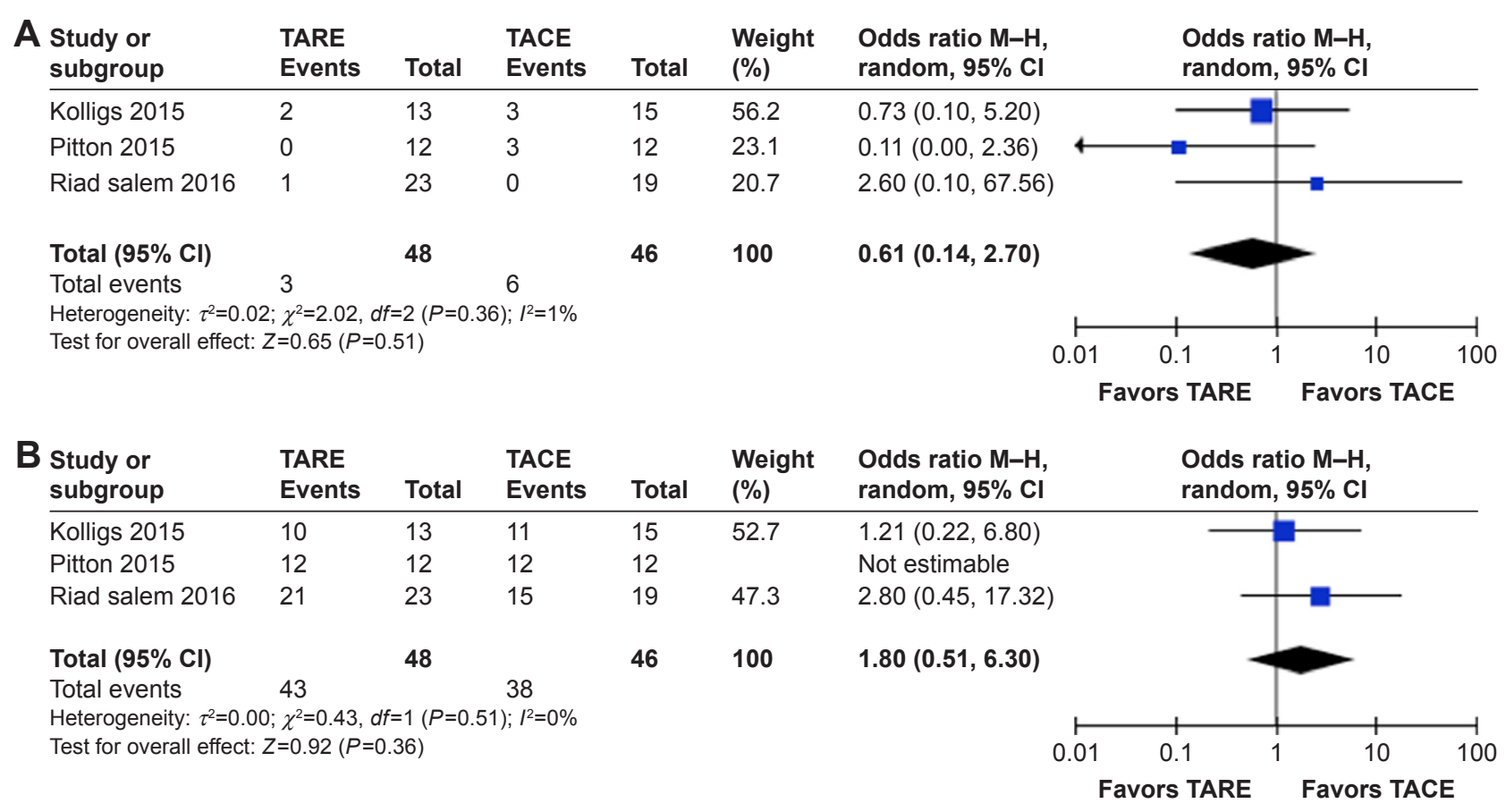

Figure 3 Forest plots of disease progression (A) and disease control rate (B).

Abbreviations: TACE, transarterial chemoembolization; TARE, transarterial radioembolization.

TARE was initially used to treat those patients who were considered suboptimal candidates for TACE due to the large tumor burden or vascular invasion. ${ }^{16,17}$ Contrary to TACE and due to the much smaller size of the beads,
TARE can be safely used in patients with portal vein occlusion or hepatofugal portal vein blood flow. ${ }^{18}$ For the same reason, it may also avoid the enhanced release of angiogenic factors, such as vascular endothelial growth factor (VEGF) 


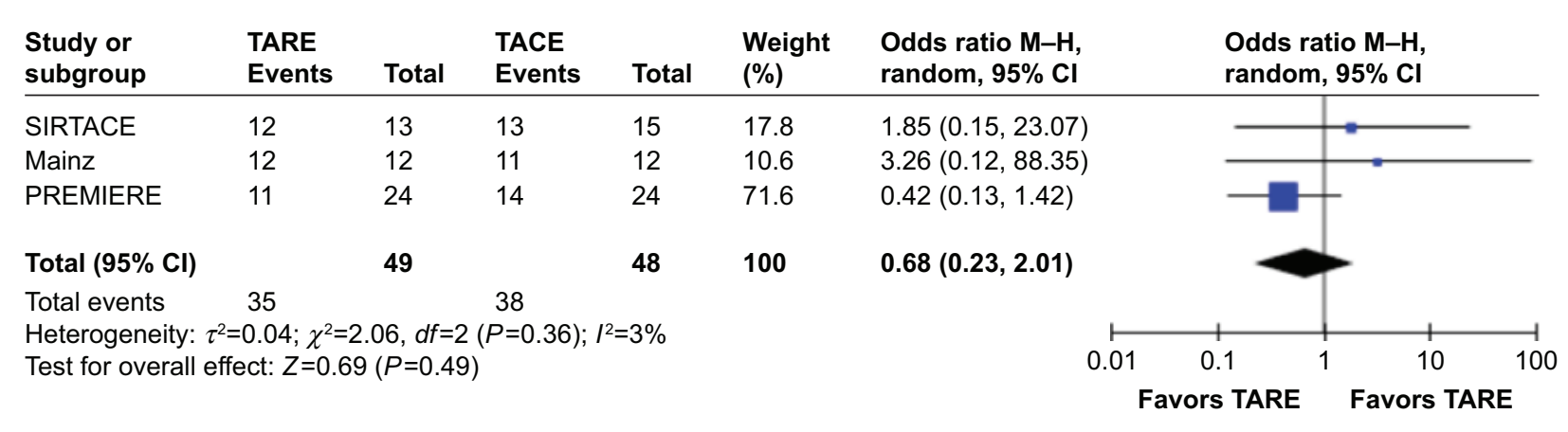

Figure 4 Forest plots of transplantation rates.

Abbreviations: TACE, transarterial chemoembolization; TARE, transarterial radioembolization.

and others, caused by the hypoxic microenvironment induced by TACE. This increase in serum VEGF levels after TACE may indeed predict a worse overall survival in TACE-treated patients. ${ }^{19}$ Finally, permanent occlusion of the feeding vessels precludes subsequent TACE procedures and contraindicates the technique when the tumors cannot be targeted in a highly selective fashion.

Other indications of TARE have been integrated in experienced centers, including radiation lobectomy to rescue patients for resection and radiation segmentectomy to achieve complete tumor necrosis when percutaneous ablation is not feasible due to tumor location. ${ }^{20}$ The indications of TACE and TARE have thus progressively overlapped and TARE may in fact compete with TACE in some treatment characteristics. For instance, while the access of cytotoxic drugs to tumor cells is hampered by tumor volume constraints in TACE, leakage from Lipiodol, and physical distance of drug-eluting particles from target tumor cells, the delivery of radiation to tumor cells in TARE is secured provided the particles reach the tumors as confirmed in the pretreatment workup.

Retrospective studies comparing single-center experience and different meta-analyses have shown no differences in outcomes. ${ }^{21}$ However, a strong patient selection bias is inherent to such studies and, therefore, the quality of the information derived from these meta-analyses is low. This is the first meta-analysis comparing TACE and TARE that analyzes a significant number of patients derived only from prospective randomized clinical trials. The analysis indicates that overall and progression-free survival at 1 year, liver transplantation rate, and disease control rate were all not statistically different between the two therapeutic groups.

The PREMIERE trial ${ }^{6}$ demonstrated a benefit of TARE compared to TACE both in terms of time to progression (HR $=0.122: 95 \%$ CI: $0.0227-0.557)$ and transplantation rate ( $54 \%$ vs $30 \%$ ), which was not observed in the other two trials, ${ }^{7,8}$ where time to progression was similar and transplantation in patients was done anecdotally. Indeed, median time to progression was not reached in the PREMIERE trial (progression rate at 1 year $>90 \%$ ) and it was 12.3 months in the Mainz trial, and progression-free survival was 3.6 months in the SIRTACE trial. These differences are probably due to the different patient characteristics. In the PREMIERE trial, ${ }^{6}$ most patients ( $75 \%$ ) were in the early stage and had unilobar involvement, while in the Mainz trial, most patients were in the intermediate stage and had bilobar tumor involvement; in the SIRTACE trial, a relevant $20 \%$ of patients were in the advanced stage due to altered performance status, which is usually associated with higher tumor burden and worse outcomes. The findings are therefore not surprising. TARE does not target micrometastases. It has already been reported that the more tumors at baseline, the higher is the chance of having early tumor progression; ${ }^{22}$ so time to progression is expected to be longer among patients at earlier stages and the impact of a highly active treatment on tumor progression is likely higher in this stage. What is less expected is that median time to new hepatic lesions was 7.3 months for TACE vs not reached for TARE in the PREMIERE trial. ${ }^{6}$ A potential explanation is that identification of progression in the targeted liver volume could be more difficult in TAREtreated livers due to the heterogeneous contrast enhancement produced by radiation in the nontumoral liver.

In terms of absolute cost, TARE is more expensive than TACE. However, no cost-effectiveness analysis has been reported so far. Such analysis should take into account the differences in procedural cost, number of procedures (in TACE, $80 \%$ of subjects receive multiple treatments, ${ }^{23}$ compared to $93 \%$ of SIRT patients receiving single treatment), ${ }^{24}$ postprocedural follow-up (less intensive for TARE), and benefit in terms of survival and HRQoL. The benefit in terms of HRQoL initially shown in a nonrandomized study ${ }^{10}$ was not confirmed in the randomized SIRTACE trial, ${ }^{8}$ reflecting the limitations of QoL evaluation. Additionally, in the various retrospective and prospective studies, as well as in meta-analyses, there was no difference in the occurrence of 
adverse events between the two procedures, with a tendency to a lower toxicity of TARE.

The main limitations of this meta-analysis are that none of the randomized controlled trials had overall survival as the main end point and, consequently, the samples are small; this is reflected in the high heterogeneity of the studies. Moreover, the target populations were not homogeneous across trials, as previously mentioned. In addition, we must also consider that in both groups, the modalities and regimens are different.

Our meta-analysis reveals that TARE and TACE have similar effects in unresectable HCC patients in terms of overall survival, disease control rate, transplantation rate, and progression rate. It is very unlikely that further trials will be conducted in unrestricted HCC populations, and our results suggest that comparative trials could better focus on specific indications, including lobar portal vein invasion, downstaging, or reduction of the dropout rate from transplant waiting lists.

\section{Disclosure}

Mercedes Iñarrairaegui has received lecture fees from Bayer Healthcare. Bruno Sangro has received lecture or consult fees from SIRTEX Medical and BTG. The authors report no other conflicts of interest in this work.

\section{References}

1. Park J-W, Chen M, Colombo M, et al. Global patterns of hepatocellular carcinoma management from diagnosis to death: the BRIDGE Study. Liver Int. 2015;35(9):2155-2166.

2. Llovet JM, Ducreux M, Lencioni R, et al; European Association For The Study Of The Liver; European Organisation For Research And Treatment Of Cancer. EASL-EORTC clinical practice guidelines: management of hepatocellular carcinoma. $J$ Hepatol. 2012;56(4):908-943.

3. Facciorusso A, Licinio R, Muscatiello N, Di Leo A, Barone M. Transarterial chemoembolization: Evidences from the literature and applications in hepatocellular carcinoma patients. World J Hepatol. 2015; 7(16):2009-2019.

4. Heimbach J, Kulik LM, Finn R, et al. AASLD guidelines for the treatment of hepatocellular carcinoma. Hepatology. 2017;67(1):358-380.

5. Sangro B, Salem R. Transarterial chemoembolization and radioembolization. Semin Liver Dis. 2014;34(4):435-443.

6. Salem R, Gordon AC, Mouli S, et al. Y90 Radioembolization Significantly Prolongs Time to Progression Compared With Chemoembolization in Patients With Hepatocellular Carcinoma. Gastroenterology. 2016;151(6):1155-1163.

7. Pitton MB, Kloeckner R, Ruckes C, et al. Randomized comparison of selective internal radiotherapy (SIRT) versus drug-eluting bead transarterial chemoembolization (DEB-TACE) for the treatment of hepatocellular carcinoma. Cardiovasc Intervent Radiol. 2015;38(2):352-360.

8. Kolligs FT, Bilbao JI, Jakobs T, et al. Pilot randomized trial of selective internal radiation therapy vs. chemoembolization in unresectable hepatocellular carcinoma. Liver Int. 2015;35(6):1715-1721.
9. Liberati A, Altman DG, Tetzlaff J, et al. The PRISMA statement for reporting systematic reviews and meta-analyses of studies that evaluate health care interventions: explanation and elaboration. J Clin Epidemiol. 2009;62(10):e1-e34.

10. Salem R, Gilbertsen M, Butt Z, et al. Increased quality of life among hepatocellular carcinoma patients treated with radioembolization, compared with chemoembolization. Clin Gastroenterol Hepatol. 2013; 11(10):1358-1365.

11. Llovet JM, Real MI, Montaña X, et al; Barcelona Liver Cancer Group. Arterial embolisation or chemoembolisation versus symptomatic treatment in patients with unresectable hepatocellular carcinoma: a randomised controlled trial. Lancet. 2002;359(9319):1734-1739.

12. Lo CM, Ngan H, Tso WK, et al. Randomized controlled trial of transarterial lipiodol chemoembolization for unresectable hepatocellular carcinoma. Hepatology. 2002;35(5):1164-1171.

13. Meyer T, Fox R, Ma YT, et al. Sorafenib in combination with transarterial chemoembolisation in patients with unresectable hepatocellular carcinoma (TACE 2): a randomised placebo-controlled, double-blind, phase 3 trial. Lancet Gastroenterol Hepatol. 2017;2(8):565-575.

14. Brown KT, Do RK, Gonen M, et al. Randomized trial of hepatic artery embolization for hepatocellular carcinoma using doxorubicin-eluting microspheres compared with embolization with microspheres alone. J Clin Oncol. 2016;34(17):2046-2053.

15. Park JW, Chen M, Colombo M, et al. Global patterns of hepatocellular carcinoma management from diagnosis to death: the BRIDGE Study. Liver Int. 2015;35(9):2155-2166.

16. Sangro B, Carpanese L, Cianni R, et al; European Network on Radioembolization with Yttrium-90 Resin Microspheres (ENRY). Survival after yttrium-90 resin microsphere radioembolization of hepatocellular carcinoma across Barcelona clinic liver cancer stages: A European evaluation. Hepatology. 2011;54(3):868-878.

17. Salem R, Lewandowski RJ, Kulik L, et al. Radioembolization results in longer time-to-progression and reduced toxicity compared with chemoembolization in patients with hepatocellular carcinoma. Gastroenterology. 2011;140(2):497-507.e2.

18. Iñarrairaegui $M$, Thurston KG, Bilbao JI, et al. Radioembolization with use of yttrium-90 resin microspheres in patients with hepatocellular carcinoma and portal vein thrombosis. J Vasc Interv Radiol. 2010; 21(8):1205-1212.

19. Shim JH, Park JW, Kim JH, et al. Association between increment of serum VEGF level and prognosis after transcatheter arterial chemoembolization in hepatocellular carcinoma patients. Cancer Sci. 2008; 99(10):2037-2044.

20. Salem R, Mazzaferro V, Sangro B. Yttrium 90 radioembolization for the treatment of hepatocellular carcinoma: biological lessons, current challenges, and clinical perspectives. Hepatology. 2013;58(6):2188-2197.

21. Sangro B, Iñarrairaegui M, Bilbao JI. Radioembolization for hepatocellular carcinoma. $J$ Hepatol. 2012;56(2):464-473.

22. Iñarrairaegui M, Martinez-Cuesta $A$, Rodríguez M, et al. Analysis of prognostic factors after yttrium-90 radioembolization of advanced hepatocellular carcinoma. Int J Radiat Oncol Biol Phys. 2010;77(5): 1441-1448.

23. Lencioni R, Llovet JM, Han G, et al. Sorafenib or placebo plus TACE with doxorubicin-eluting beads for intermediate stage HCC: The SPACE trial. J Hepatol. 2016;64(5):1090-1098.

24. Sangro B, Carpanese L, Cianni R, et al; European Network on Radioembolization with Yttrium-90 Resin Microspheres (ENRY). Survival after yttrium-90 resin microsphere radioembolization of hepatocellular carcinoma across Barcelona clinic liver cancer stages: a European evaluation. Hepatology. 2011;54(3):868-878. 


\section{Supplementary material}

A
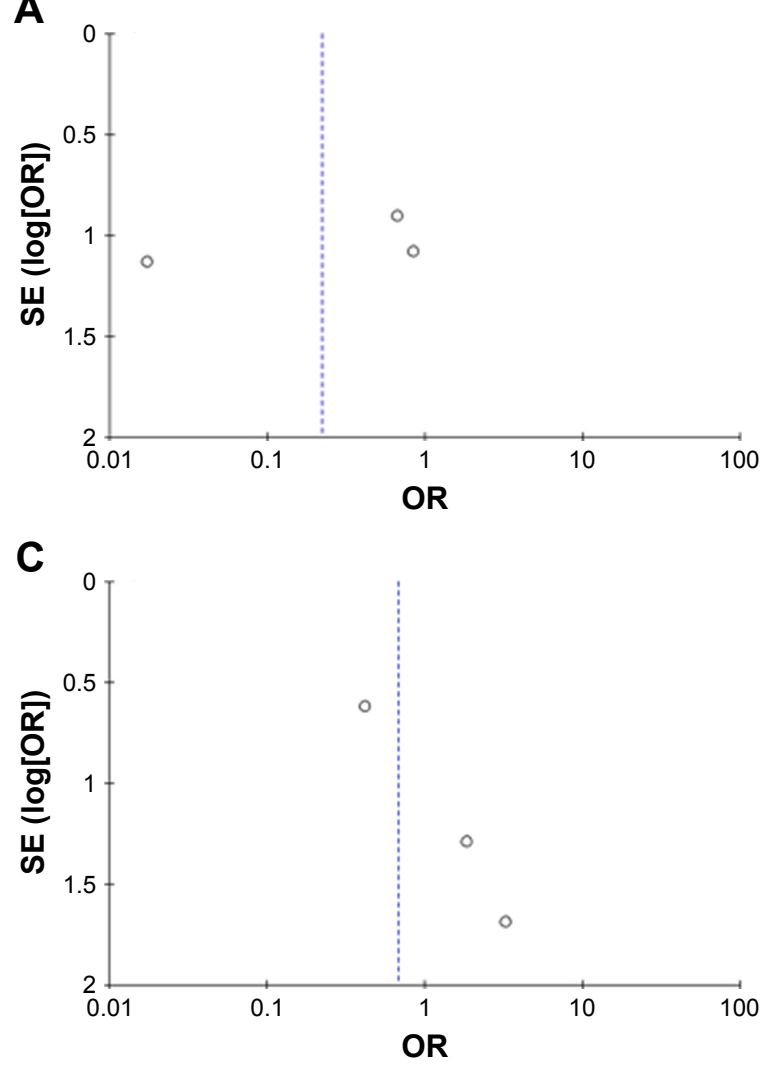

B

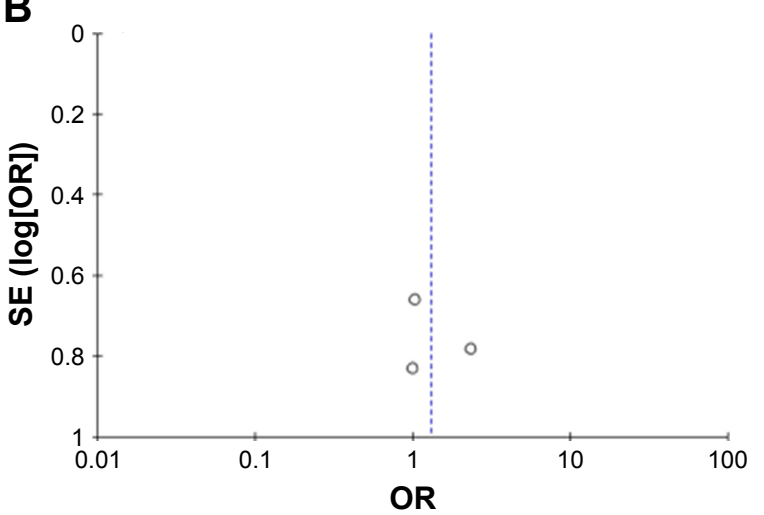

D

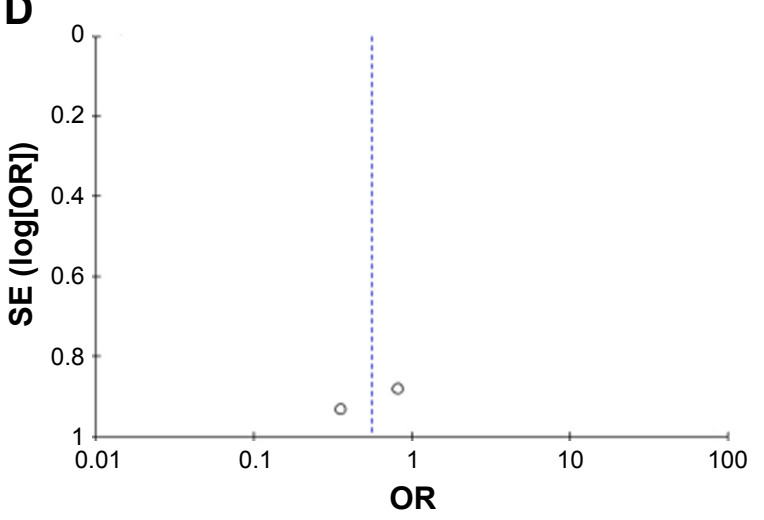

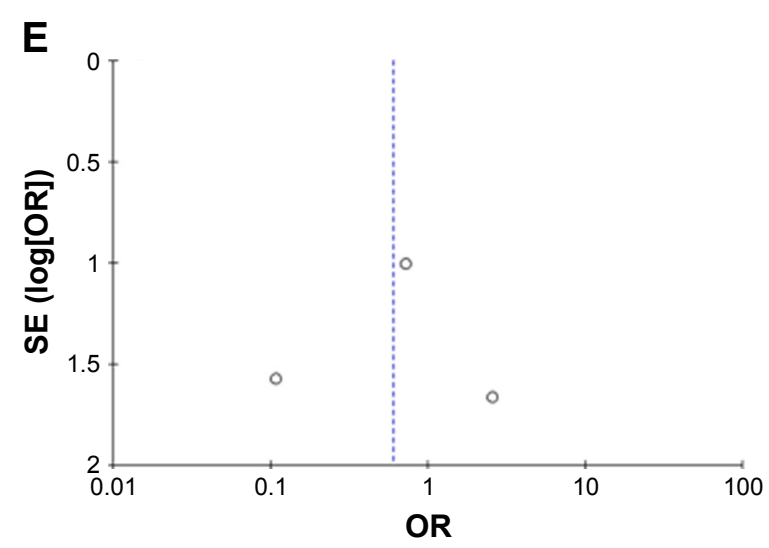

Figure SI Funnel plot of the study.

Notes: Funnel plot of progression free survival I year (A); overall survival I year (B); transplant rate (C); disease control rate (D); and progression disease (E).

\section{Publish your work in this journal}

OncoTargets and Therapy is an international, peer-reviewed, open access journal focusing on the pathological basis of all cancers, potential targets for therapy and treatment protocols employed to improve the management of cancer patients. The journal also focuses on the impact of management programs and new therapeutic agents and protocols on patient perspectives such as quality of life, adherence and satisfaction. The manuscript management system is completely online and includes a very quick and fair peer-review system, which is all easy to use. Visit http://www.dovepress.com/testimonials.php to read real quotes from published authors. 